\title{
Práticas de matriciamento em saúde mental desenvolvidas na atenção primária à saúde
}

\author{
Mental health matrix support practices developed in primary health care \\ Prácticas de apoyo a la matriz de salud mental desarrolladas en la atención primaria de \\ salud
}

Helio Alves da Silva ${ }^{1 *}$, Álvaro Ataide Landulfo Teixeira ${ }^{2}$, Laudileyde Rocha Mota ${ }^{1}$, Letícia Oliveira Silva $^{3}$, Jeniffer Silva Oliveira ${ }^{3}$, Luzete Celestino da Silva Gonçalves ${ }^{3}$, Isabella Batista Vieira ${ }^{3}$, Alcina Mendes Brito ${ }^{4}$, Kelly Karoline Eugênio ${ }^{3}$, Aparecida Samanta Lima Gonçalves ${ }^{1}$.

\section{RESUMO}

Objetivo: Conhecer as práticas de matriciamento em saúde mental desenvolvidas na atenção primária à saúde Métodos: Trata-se de uma revisão integrativa de literatura realizada por meio das bases de dados secundários Scielo, Lilacs e Biblioteca Virtual em Saúde. Resultados: Foram encontrados 42 artigos na Biblioteca Virtual em Saúde, 16 na Scielo e 33 na Lilacs, após aplicação dos critérios de inclusão e exclusão foram selecionados 13 artigos para a amostra final. Algumas barreiras foram indicadas para realização do matriciamento: fragilização da política nacional de atenção básica, heterogeneidade de concepções e modelos de prática, ausência de suporte das gestões e gerência dos serviços, ausência de periodicidade do processo e pouca interferência na organização dos fluxos na rede Rede de Atenção Psicossocial, agenda diversas e problemas entre a Estratégia Saúde da Família e equipes apoiadoras, pouca presença do psiquiatra nas ações de apoio matricial, lógica inserida no modelo ambulatorial com foco na prática de encaminhamento e marcação de consultas, bem como a maneira de implantação da proposta em muitas cidades do país. Considerações finais: $\mathrm{Na}$ análise das práticas de apoio matricial desenvolvidas nos cenários da atenção primária à saúde em caráter nacional, reconhece-se um processo embrionário, ainda a ser intensificado e fortalecido.

Palavras-chave: Saúde mental, Gestão em saúde, Atenção primária à saúde.

\begin{abstract}
Objective: To know the mental health matrix support practices developed in primary health care Methods: It is an integrative literature review carried out through the secondary databases Scielo, Lilacs and Virtual Health Library. Results: 42 articles were found at the Virtual Health Library, 16 at Scielo and 33 at Lilacs, after applying the inclusion and exclusion criteria, 13 articles were selected for the final sample. Some barriers were indicated for matrix support: weakening of the national primary care policy, heterogeneity of conceptions and models of practice, absence of support from management and service management, absence of periodicity of the process and little interference in the organization of flows in the network of Psychosocial Care, diverse agenda and problems between the Family Health Strategy and support teams, little presence of the psychiatrist in matrix support actions, logic inserted in the outpatient model focused on the practice of referral and scheduling consultations, as well as the way of implantation of the proposal in many cities in the country. Final considerations: In the analysis of matrix support practices developed in primary health care scenarios on a national basis, an embryonic process is recognized, yet to be intensified and strengthened.
\end{abstract}

Keywords: Mental health, Health management, Primary health care.

\section{RESUMEN}

Objetivo: Conocer las prácticas de apoyo a la matriz de salud mental desarrolladas en la atención primaria de salud. Métodos: Se trata de una revisión integradora de la literatura realizada a través de las bases de datos secundarias Scielo, Lilacs y Biblioteca Virtual en Salud. Resultados: 42 artículos fueron encontrados en la Biblioteca Virtual en Salud, 16 en Scielo y 33 en Lilacs, luego de aplicar los criterios de inclusión y exclusión, se seleccionaron 13 artículos para la muestra final. Se señalaron algunas barreras para el soporte matricial: debilitamiento de la política nacional de atención primaria, heterogeneidad de concepciones y modelos de práctica, falta de apoyo desde la gestión y gestión del servicio, ausencia de periodicidad del proceso y poca interferencia en la organización de flujos en el red de Atención Psicosocial, agenda y problemática diversa entre la Estrategia Salud

\footnotetext{
${ }^{1}$ Faculdades Santo Agostinho (FASA), Montes Claros - MG. *E-mail: scmenfermagem9@gmail.com

2 Faculdades Integradas Pitágoras (PITÁGORAS), Montes Claros - MG.

${ }^{3}$ Faculdades Unidas do Norte de Minas Gerais (FUNORTE), Montes Claros - MG.

${ }^{4}$ Pontifícia Universidade Católica de Minas Gerais (PUCMINAS), Montes Claros - MG.
} 
de la Familia y los equipos de apoyo, poca presencia del psiquiatra en las acciones de apoyo matricial, lógica insertada en el modelo ambulatorio con enfoque en la práctica de derivación y programación de consultas, así como la forma de implantación de la propuesta en muchas ciudades del país. Consideraciones finales: En el análisis de las prácticas de soporte matricial desarrolladas en escenarios de atención primaria de salud a nivel nacional, se reconoce un proceso embrionario, aún por intensificar y fortalecer.

Palabras clave: Salud mental, Gestión de la salud, Atención primaria de salud.

\section{INTRODUÇÃO}

Após três décadas do processo motivador da Reforma Psiquiátrica, foram poucas as transformações associadas à prática dos profissionais de saúde, ainda que tenham ocorrido a publicação de leis e portarias que regulamentaram estratégias nos diferentes níveis de assistência. A principal aspiração da reforma psiquiátrica é a desinstitucionalização, que almeja não apenas a desconstrução do manicômio, mas o resgate do fenômeno da loucura por meio da produção de novas definições, cuidados e conhecimentos. Nos últimos anos, o Ministério da Saúde iniciou o processo de inserção da área da saúde mental na Atenção Primária à Saúde com diretrizes para a estruturação das ações, sendo estas baseadas em algumas noções e definições como diálogo entre os níveis, acolhimento, responsabilização, construção de vínculos e integralidade da assistência (ALMEIDA DL, et al., 2019).

A elevada prevalência dos transtornos mentais em contraponto com a carência de serviços é um obstáculo para a saúde pública. As estimativas globais assinalam a necessidade de melhoria nos sistemas de saúde, já que a taxa dos que necessitam de tratamento e não são tratados apresenta elevados índices, sobretudo, nos países emergentes (IGLESIAS A e AVELLAR LZ, 2014).

Por um longo período na história, a hospitalização, o enclausuramento, a exclusão e o isolamento foram tomados como única forma de lidar com a "loucura". As pessoas em sofrimento psíquico, "desprovidas" de sua potência produtiva, em período de crescimento e fortalecimento do capitalismo, eram depositadas nos manicômios, onde sofriam com a violação explícita de sua integridade física, moral e psíquica. Frente ao contexto de movimentação política e social para a democratização do Brasil, esse modelo hospitalocêntrico e medicalizante passou a ser fortemente criticado, favorecendo a reestruturação do modelo assistencial à saúde mental, preconizado pelo Movimento da Reforma Psiquiátrica (IGLESIAS A e AVELLAR LZ, 2014).

A reforma psiquiátrica brasileira surge na década de 1980, em consonância com diversos outros movimentos mundiais que vinham ocorrendo à época, na busca de denunciar práticas asilares opressoras e que infligiram os direitos humanos dos usuários no campo da saúde mental. O referido movimento é um processo ético, político e social, que busca pautar uma série de ressignificações no modelo de cuidado em saúde mental, afetando trabalhadores, usuários, familiares, gestores, além de significativa interferência no território e no imaginário das pessoas nele inseridas. O cuidado passa a ter como premissa a defesa da vida e a percepção dos usuários como cidadãos pertencentes à comunidade. Para tanto, é necessário considerar as conexões relacionais que envolvem o sujeito que é cuidado e entendê-las como produtoras de vida (FERREIRA TPS, et al., 2017).

O movimento tem como foco a desinstitucionalização, que não se restringe a desospitalização, já que a retirada destas pessoas dos hospitais não acarreta numa mudança automática das atitudes de intolerância frente à diferença. A desinstitucionalização implica em mudança cultural, política, social, ideológica e ética no modo de lidar com o sofrimento psíquico. Para tanto, requer a criação de condições para que estas pessoas recém-saídas dos hospitais psiquiátricos, e os novos pacientes, sejam inseridos socialmente, acolhidos e tratados em seu sofrimento (IGLESIAS A e AVELLAR LZ, 2014).

Nesse novo modelo de assistência em saúde mental, a atenção básica surge como eixo estruturante do sistema, pois, além de ser 'porta de entrada', gerencia os encaminhamentos, coordena e integra o trabalho realizado por outros níveis de atenção, outros equipamentos ou por terceiros e acompanha, de maneira longitudinal, a saúde do paciente durante a vida (GAZIGNATO ECS e SILVA CRC, 2014). As ações de saúde mental desenvolvidas no âmbito da Atenção Básica exercem papel fundamental ao contribuir com os saberes para a ampliação do potencial resolutivo das equipes, mediante a realização do apoio matricial (AM) ou matriciamento de saúde mental que tem por objetivo superar a lógica da especialização e da fragmentação do trabalho da própria área de saúde mental (BRASIL, 2008). 
O apoio matricial pode ser definido como um arranjo organizacional que surge com o objetivo de ampliar a capacidade de resolubilidade das ações de saúde, ao propor uma reformulação no modo de organização dos serviços e relações horizontais entre as especialidades que passam a oferecer apoio técnico horizontal às equipes interdisciplinares de atenção primária, favorecendo assim a conexão em rede (JORGE MSB, et al., 2013)

O apoio matricial ou matriciamento, assim, pode ser entendido como um arranjo organizacional construído no cenário brasileiro e que, sempre baseado no contexto real do território, possibilita uma nova maneira de se construir saúde por meio da troca de saberes e da interlocuação dialógica existente entre as equipes de saúde da família referência e os profissionais que figuram como matriciadores (CHIAVERINI DH, 2011). Ressalta-se que, em relação à saúde mental, o papel do matriciador frequentemente é exercida por psicólogos, psiquiatras, terapeutas ocupacionais, assistentes sociais e enfermeiros, sobretudo, vinculados ao Centro de Atenção Psicossocial (GONÇALVES RC e PERES RS, 2018).

Ainda que o Ministério da Saúde oriente de forma inequívoca que os serviços de atenção básica a saúde estruturem a assistência em saúde mental tendo como base o AM, o diálogo entre os profissionais e os diferentes níveis hierárquicos acontece, frequentemente, de maneira incoerente (CHIAVERINI DH, 2011). Nesse contexto, o objetivo do estudo foi conhecer as práticas de matriciamento em saúde mental desenvolvidas na atenção primária à saúde.

\section{MÉTODOS}

Realizou-se um estudo de revisão integrativa de literatura. A revisão integrativa de literatura objetiva reunir e sistematizar os desfechos de pesquisas sobre determinada temática de maneira tal que possa contribuir para o aprofundamento e entendimento da temática selecionada (MENDES KDS, et al., 2008).

A presente revisão estruturou-se a partir das orientações para construção da revisão integrativa de literatura, na qual se apresentam nas seguintes fases: 1) Elaboração da pergunta norteadora; 2) Busca na literatura; 3) Coleta de dados; 4) Análise crítica dos estudos incluídos; 5) Discussão dos resultados e 6) Apresentação da revisão integrativa. A questão norteadora estabelecida foi: Quais as práticas de matriciamento em saúde mental desenvolvidas na atenção primária à saúde no cenário brasileiro? (SOUZA MC, et al., 2010).

A coleta de dados foi conduzida em durante o segundo semestre de 2020 a partir das bases de dados secundários Scientific Electronic Library Online (Scielo), Literatura Latino-Americana e do Caribe em Ciências da Saúde (LILACS) e a Biblioteca Virtual em Saúde (BVS). Para guiar a coleta de dados utilizou-se instrumento construído e validado com as seguintes variáveis: dados de identificação do artigo (título, autores, nome do periódico, ano de publicação, volume e número), tipo de estudo, local de estudo, objetivo do estudo, eixo temático, classificação Qualis/Capes, periódico de publicação, nível de evidência, desfechos, dentre outros.

Foram utilizados como descritores "Atenção Primária à Saúde", "Saúde Mental", "Apoio Matricial" e "Matriciamento", foi utilizado o operador booleano "and" para busca. Os critérios de inclusão estabelecidos foram: artigos completos, publicados no idioma nacional, devido ao objetivo proposto de conhecer a realidade brasileira, o corte temporal foi de cinco anos. Foram excluídas teses, dissertações, cartilhas, trabalhos de conclusão de curso e monografias, além de artigos que não abordassem de forma relevante a temática do estudo.

\section{RESULTADOS}

Inicialmente foram identificados 91 artigos que poderiam ser analisados para a construção do trabalho, após a triagem inicial foi realizado a análise dos títulos e resumos dos artigos, sendo aplicados os critérios de exclusão e inclusão, sendo excluídos ainda os artigos em duplicidade, o que resultou na amostra final de 13 artigos. Para melhor visualização das pesquisas incluídas na presente investigação, foi construído um quadro com as seguintes variáveis: ano, autores, título do artigo, período de publicação, delineamento do estudo, objetivo geral e principais desfechos. 
Quadro 1 - Artigos selecionados para análise.

\begin{tabular}{|c|c|c|c|c|c|}
\hline Autor(es)/Ano & Título & Periódico & Delineamento & Objetivo & Desfecho \\
\hline $\begin{array}{l}\text { Belottia M e } \\
\text { Lavrador MCC } \\
\quad(2016) .\end{array}$ & $\begin{array}{l}\text { A prática do apoio matricial e } \\
\text { os seus efeitos na Atenção } \\
\text { Primária à Saúde. }\end{array}$ & $\begin{array}{l}\text { Cad. Ter. Ocup. } \\
\text { UFSCar. }\end{array}$ & Relato de experiência. & $\begin{array}{l}\text { Identificar os efeitos que os encontros de } \\
\text { matriciamento provocado no território de } \\
\text { adstringência das equipes da Estratégia } \\
\text { Saúde da Família acompanhadas. }\end{array}$ & $\begin{array}{l}\text { O Apoio Matricial é uma ferramenta capaz } \\
\text { de promover uma interlocução entre os } \\
\text { serviços de saúde mental e a Atenção } \\
\text { Primária à Saúde. }\end{array}$ \\
\hline $\begin{array}{l}\text { Vasconcelos SM } \\
\text { e Barbosa BFV } \\
\text { (2019). }\end{array}$ & $\begin{array}{l}\text { Conhecimento de gestores e } \\
\text { profissionais da rede de } \\
\text { atenção psicossocial sobre } \\
\text { matriciamento em saúde } \\
\text { mental. }\end{array}$ & Cienc Cuid Saude. & $\begin{array}{l}\text { Trata-se de um estudo } \\
\text { descritivo de abordagem } \\
\text { qualitativa. }\end{array}$ & $\begin{array}{l}\text { Identificar o conhecimento dos gestores e } \\
\text { profissionais de saúde de um município do } \\
\text { sertão pernambucano sobre o apoio } \\
\text { matricial em saúde mental. }\end{array}$ & $\begin{array}{l}\text { As lacunas de abordagem e discussão } \\
\text { sobre apoio matricial em saúde mental na } \\
\text { formação acadêmica de gestores e } \\
\text { profissionais de saúde influenciam } \\
\text { negativamente o seu conhecimento } \\
\text { conceitual sobre o tema e a sua } \\
\text { habilidade de implementar ferramentas de } \\
\text { matriciamento em saúde mental. }\end{array}$ \\
\hline $\begin{array}{l}\text { Salvador BD e Pio } \\
\text { MADD (2016). }\end{array}$ & $\begin{array}{l}\text { Apoio Matricial e Capsi: } \\
\text { desafios do cenário na } \\
\text { implantação do } \\
\text { matriciamento em saúde } \\
\text { mental. }\end{array}$ & Saúde Debate. & $\begin{array}{l}\text { Estudo do tipo qualitativo e } \\
\text { exploratório descritivo. }\end{array}$ & $\begin{array}{l}\text { Explorar o conhecimento e a experiência de } \\
\text { uma equipe de Centro de Atenção } \\
\text { Psicossocial Infantil (Capsi) acerca da } \\
\text { estratégia do Apoio Matricial (AM), com a } \\
\text { participação de cinco profissionais da } \\
\text { equipe de referência e quatro residentes } \\
\text { multiprofissionais. }\end{array}$ & $\begin{array}{l}\text { Não se chegou a uma definição clara } \\
\text { sobre o tema e não se reconheceu uma } \\
\text { experiência com AM, tendo dificuldades } \\
\text { para implantar esta estratégia dentro das } \\
\text { ações de cuidado em saúde mental. }\end{array}$ \\
\hline $\begin{array}{l}\text { Garcia DVG, et al. } \\
(2017) .\end{array}$ & $\begin{array}{l}\text { Apoio matricial na atenção à } \\
\text { saúde mental em uma } \\
\text { regional de saúde, Paraná, } \\
\text { Brasil. }\end{array}$ & $\begin{array}{l}\text { Revista Saúde e } \\
\text { Pesquisa. }\end{array}$ & $\begin{array}{c}\text { Estudo exploratório e } \\
\text { descritivo, com abordagem } \\
\text { qualitativa. }\end{array}$ & $\begin{array}{l}\text { Analisar os limites e possibilidades do apoio } \\
\text { matricial em saúde mental na Atenção } \\
\text { Básica, segundo a percepção de gestores e } \\
\text { profissionais de saúde em uma regional de } \\
\text { saúde, Paraná, Brasil. }\end{array}$ & $\begin{array}{l}\text { As equipes de apoio matricial precisam } \\
\text { assumir a responsabilidade de suporte } \\
\text { técnico à atenção básica principalmente } \\
\text { no acolhimento às necessidades de } \\
\text { saúde mental. }\end{array}$ \\
\hline $\begin{array}{l}\text { Lima M e } \\
\text { Dimenstein M } \\
\quad(2016) .\end{array}$ & $\begin{array}{l}\text { O apoio matricial em saúde } \\
\text { mental: uma ferramenta } \\
\text { apoiadora da atenção à } \\
\text { crise. }\end{array}$ & Interface. & $\begin{array}{l}\text { Estudo qualitativo como } \\
\text { uma Pesquisa Intervenção. }\end{array}$ & $\begin{array}{l}\text { Conhecer apoio matricial em saúde mental } \\
\text { como uma ferramenta apoiadora da } \\
\text { atenção à crise. }\end{array}$ & $\begin{array}{l}\text { O matriciamento se revelou ferramenta } \\
\text { apoiadora da atenção à crise, ao prevenir } \\
\text { situações de crise ou abordá-las antes } \\
\text { que se agravem, evitando manejos mais } \\
\text { invasivos como: a utilização da polícia, } \\
\text { uso da força física, conduções } \\
\text { involuntárias para a emergência, } \\
\text { internação hospitalar e para o CAPS. }\end{array}$ \\
\hline $\begin{array}{l}\text { Freitas FN e } \\
\text { Passos FCl } \\
\text { (2018). }\end{array}$ & $\begin{array}{l}\text { Apoio matricial em saúde } \\
\text { mental no SUS de Belo } \\
\text { Horizonte: Perspectiva dos } \\
\text { trabalhadores. }\end{array}$ & Trab. Educ. Saúde. & $\begin{array}{c}\text { Trata-se de pesquisa } \\
\text { empírica. }\end{array}$ & $\begin{array}{l}\text { Analisar a prática do apoio matricial (AM) } \\
\text { em saúde mental mediante o estudo de } \\
\text { caso da experiência da cidade de Belo } \\
\text { Horizonte, em Minas Gerais. }\end{array}$ & $\begin{array}{l}\text { O apoio matricial tem transformado a } \\
\text { relação de trabalho nas unidades básicas } \\
\text { de saúde no sentido da } \\
\text { corresponsabilização e do trabalho } \\
\text { integrado entre as equipes. }\end{array}$ \\
\hline
\end{tabular}




\begin{tabular}{|c|c|c|c|c|c|}
\hline Autor(es)/Ano & Título & Periódico & Delineamento & Objetivo & Desfecho \\
\hline $\begin{array}{c}\text { Iglesias A e } \\
\text { Avellar ZL (2016). }\end{array}$ & $\begin{array}{l}\text { As contribuições dos } \\
\text { Psicólogos para o } \\
\text { Matriciamento em Saúde } \\
\text { Mental. }\end{array}$ & $\begin{array}{l}\text { Psicologia: Ciência e } \\
\text { Profissão. }\end{array}$ & Pesquisa qualitativa. & $\begin{array}{l}\text { Investigar as concepções do psicólogo da } \\
\text { atenção básica sobre o apoio matricial em } \\
\text { saúde mental. }\end{array}$ & $\begin{array}{l}\text { Alguns psicólogos apresentaram } \\
\text { dificuldades em afirmar outra prática que } \\
\text { não aquela voltada ao atendimento } \\
\text { ambulatorial individual, mas também } \\
\text { outros conseguiram estabelecer parcerias } \\
\text { diversas para o cuidado em saúde. }\end{array}$ \\
\hline $\begin{array}{l}\text { Treichel SAC, et } \\
\text { al. (2019). }\end{array}$ & $\begin{array}{l}\text { Impasses e desafios para } \\
\text { consolidação e efetividade } \\
\text { do apoio matricial em saúde } \\
\text { mental no Brasil. }\end{array}$ & Interface. & $\begin{array}{c}\text { Foi realizada uma revisão } \\
\text { bibliográfica integrativa. }\end{array}$ & $\begin{array}{l}\text { Revisar a bibliografia nacional dos últimos } \\
\text { dez anos a fim de identificar os impasses e } \\
\text { desafios para consolidação e efetividade do } \\
\text { apoio matricial em Saúde Mental na } \\
\text { Atenção Primária. }\end{array}$ & $\begin{array}{l}\text { Há necessidade de delineamentos claros } \\
\text { para prática de matriciamento; } \\
\text { investimento maciço em formação e } \\
\text { capacitação dos profissionais; e criação } \\
\text { de espaços institucionalizados com } \\
\text { encontros sistemáticos dos profissionais } \\
\text { para discussão dos casos e avaliação } \\
\text { conjunta do andamento das atividades. }\end{array}$ \\
\hline $\begin{array}{l}\text { Gonçalves CR e } \\
\text { Peres SR (2018). }\end{array}$ & $\begin{array}{l}\text { Matriciamento em saúde } \\
\text { mental: obstáculos, } \\
\text { caminhos e resultados. }\end{array}$ & Revista da SPAGESP. & Revisão da literatura. & $\begin{array}{l}\text { Estabelecer um panorama da produção } \\
\text { científica brasileira sobre o matriciamento } \\
\text { em saúde mental, com foco na articulação } \\
\text { entre a atenção primária e os CAPSs. }\end{array}$ & $\begin{array}{l}\text { O matriciamento pode contribuir de } \\
\text { diferentes maneiras para a qualificação } \\
\text { da assistência em saúde mental, mas } \\
\text { ainda há uma série de obstáculos, alguns } \\
\text { mais evidentes e outros mais velados, a } \\
\text { serem transpostos nesta direção. }\end{array}$ \\
\hline $\begin{array}{c}\text { Silva ACJL, et al. } \\
\text { (2019). }\end{array}$ & $\begin{array}{l}\text { A contribuição do apoiador } \\
\text { matricial na superação do } \\
\text { modelo psiquiátrico } \\
\text { tradicional. }\end{array}$ & Psicol. Estud. & Relato de experiência. & $\begin{array}{l}\text { Relatar o processo de trabalho do apoiador } \\
\text { matricial para o cuidado em saúde mental } \\
\text { na atenção básica. }\end{array}$ & $\begin{array}{l}\text { As atividades de matriciamento } \\
\text { potencializaram a atuação das equipes da } \\
\text { Atenção Básica para as demandas de } \\
\text { saúde mental no território. }\end{array}$ \\
\hline $\begin{array}{c}\text { Santos MA e et al } \\
(2020) .\end{array}$ & $\begin{array}{l}\text { O matriciamento em saúde } \\
\text { mental como dispositivo para } \\
\text { a formação e gestão do } \\
\text { cuidado em saúde. }\end{array}$ & $\begin{array}{l}\text { Physis: Revista de } \\
\text { Saúde Coletiva. }\end{array}$ & Pesquisa qualitativa. & $\begin{array}{l}\text { Refletir a partir da pesquisa realizada no } \\
\text { município de Piraí-RJ sobre o processo de } \\
\text { matriciamento e suas implicações para a } \\
\text { gestão do cuidado em saúde. }\end{array}$ & $\begin{array}{l}\text { O matriciamento é um dispositivo para a } \\
\text { produção de subjetividades para a gestão } \\
\text { do cuidado em saúde. }\end{array}$ \\
\hline $\begin{array}{c}\text { Iglesias A e } \\
\text { Avellar ZL (2019). }\end{array}$ & $\begin{array}{l}\text { Matriciamento em Saúde } \\
\text { Mental: práticas e } \\
\text { concepções trazidas por } \\
\text { equipes de referência, } \\
\text { matriciadores e gestores. }\end{array}$ & $\begin{array}{l}\text { Ciência \& Saúde } \\
\text { Coletiva. }\end{array}$ & $\begin{array}{c}\text { Pesquisa de abordagem } \\
\text { qualitativa. }\end{array}$ & $\begin{array}{l}\text { Analisar o matriciamento em saúde mental } \\
\text { a partir das práticas e concepções trazidas } \\
\text { pelas equipes de referência, equipes } \\
\text { matriciais e gestores a respeito da temática. }\end{array}$ & $\begin{array}{l}\text { Há necessidade de criação, } \\
\text { sistematização e fortalecimento de } \\
\text { espaços de diálogo entre equipes de } \\
\text { referência, equipes matriciais e gestores } \\
\text { para a consolidação do matriciamento. }\end{array}$ \\
\hline
\end{tabular}

Fonte: Silva HA, et al., 2021. 


\section{DISCUSSÃO}

O diálogo entre os serviços de assistência à saúde mental ocorre, frequentemente, de maneira precária e assistemática, comumente através de encaminhamentos escritos, como por exemplo, solicitações de parecer e registros de contra referência que não permitem uma resposta efetiva. De forma, tradicional, os serviços de saúde se estruturam de maneira vertical (hierárquica), com uma diferenciação de autoridade entre o ator que faz o encaminhamento e o profissional que o recepta, existindo um processo de transferência de responsabilidades ao fazer o encaminhamento (CHIAVERINI DH, 2011; FORTES S, et al., 2014).

A nova proposta integradora visa transformar a lógica tradicional dos sistemas de saúde: encaminhamentos, referências e contra referências, protocolos e centros de regulação. Os efeitos burocráticos e pouco dinâmicos dessa lógica tradicional podem vir a ser atenuados por ações horizontais que integrem os componentes e seus saberes nos diferentes níveis assistenciais. Em contraponto a essa lógica reducionista, foi elaborada a proposta do matriciamento, assim, na horizontalização decorrente do processo de matriciamento, o sistema de saúde se reestrutura em dois tipos de equipes: a) Equipe de referência; b) Equipe de apoio matricial. Esse apoio matricial, formulado na gestão da clínica, tem estruturado no país um tipo de cuidado colaborativo entre a saúde mental e a atenção primária (CHIAVERINI DH, 2011).

O Matriciamento ou também denominado apoio matricial é uma nova modalidade de produção de saúde, nessa modalidade de cuidado, duas ou mais equipes, em um contexto de construção conjunta, elaboram uma proposta de cuidado pedagógico-terapêutica (FORTES S, et al., 2014). O matriciamento deve proporcionar a retaguarda especializada da assistência, assim como um suporte técnico-pedagógico, um vínculo interpessoal e o apoio institucional no processo de construção coletiva de matriciamento, integrando, assim saúde mental e atenção primária em um modelo de cuidados colaborativos na construção de projetos terapêuticos junto à população. Assim, também se diferencia da supervisão, pois o matriciador pode participar ativamente do projeto terapêutico. O Matriciamento constitui-se numa ferramenta de transformação, não só do processo de saúde e doença, mas de toda a realidade das equipes e comunidades (CHIAVERINI DH, 2011).

A inserção de práticas de saúde mental na atenção básica evidencia a busca pela regionalização e redirecionamento do cuidado, numa perspectiva de atenção integral e humanizada aos sujeitos, em articulação com profissionais e serviços já inseridos nos territórios. A Organização Mundial da Saúde, por meio da Declaração de Caracas, postula a reestruturação da atenção psiquiátrica por meio da Atenção Primária em Saúde (APS) como promotora de modelos alternativos centrados nas comunidades e em suas redes sociais, em conformidade com os direitos humanos (GAZIGNATO ECS e SILVA CRC, 2014).

O Ministério da Saúde propôs a estratégia do Apoio Matricial (AM), ou matriciamento em saúde mental, para facilitar o direcionamento dos fluxos na rede, promovendo a articulação entre os equipamentos de saúde mental e a ESF. Segundo a coordenação de saúde mental, no documento apresentado à Conferência Regional de Reforma dos Serviços de Saúde Mental, o AM se constitui em um arranjo organizacional que viabiliza o suporte técnico em áreas específicas para equipes responsáveis pelo desenvolvimento de ações básicas de saúde (BRASIL, 2008).

Ademais, o matriciamento se afirma como uma estratégia de produção de novas práticas em saúde mental também junto às comunidades, nos territórios onde os indivíduos vivem e convivem, pela sua proposta de momentos produtivos, sistemáticos e interativos entre as equipes multiprofissionais da atenção primária à saúde e equipes de saúde mental (IGLESIAS A, AVELLAR LZ, 2019).

Em uma nova roupagem em rede, o matriciamento assume o papel de uma estratégia que possibilita a integração entre os dispositivos de atenção à saúde e os demais níveis internos e externos que compõem essa rede (MACHADO DKS e CAMATTA MW, 2013). O matriciamento é uma tecnologia que estrutura o processo de trabalho e a assistência com o objetivo de possibilitar um processo de horizontalização das diversas especialidades, permitindo que estas façam parte de todo o cotidiano das equipes de saúde (WETZEL C, et al., 2014).

Nesse arranjo, a equipe de saúde mental compartilha alguns casos com as equipes de atenção básica. Esse compartilhamento se produz em forma de corresponsabilização pelos casos, que podem se efetivar 
através de discussões conjuntas de casos, intervenções conjuntas junto às famílias e comunidades ou em atendimentos conjuntos, e também na forma de supervisão e capacitação. O matriciamento em saúde mental reduz a lógica dos encaminhamentos, uma vez que, a maioria dos casos de saúde mental podem ser resolvidos na atenção primária, além disso, o apoio matricial em saúde mental oferece um suporte técnico especializado aos profissionais da atenção básica, qualificando e capacitando suas ações. Esse trabalho interdisciplinar favorece a troca de saberes entre os profissionais, dessa forma, oportuniza ao paciente um atendimento e acompanhamento de qualidade (BRASIL, 2008).

A atenção primária tem como um dos seus norteadores permitir o primeiro acesso dos indivíduos ao sistema de saúde, incluindo daqueles que procuram cuidados associados a saúde mental. Nesse tipo de assistência, o cuidado é desenvolvido em um território geograficamente delimitado, permitindo aos profissionais de saúde uma aproximação para compreender a história de vida dos indivíduos e de seus vínculos com a comunidade e território onde residem, bem como outros dispositivos de suas realidades de vida. Compreende-se assim, que a assistência em saúde mental na atenção primária é bastante estratégica pelo fácil acesso das equipes aos indivíduos e vice-versa (BRASIL, 2013; CHIAVERINI DH, 2011).

Essa assistência aos indivíduos com diagnóstico dos transtornos mentais nas Unidades Básicas de Saúde possibilita que os profissionais da atenção primária maximizem seus conhecimentos sobre o cuidado em saúde mental, em um prisma de humanização e integralidade, assim como possibilita que intensifiquem os vínculos com os indivíduos e famílias, facilitando, assim, a abordagem dos casos, a corresponsabilização e a resolutividade. Como resultado, os encaminhamentos inoportunos aos serviços especializados e os gastos ao sistema de saúde são reduzidos (QUINDERÉ PHD, et al., 2013; GAZIGNATO ECS e CASTRO CRS, 2014; NETO MRG, et al., 2014).

Em razão do apoio matricial ser uma estratégia nova em saúde mental organizada entre diferentes atores pressupõe-se que há maneiras peculiares de sua construção em decorrência dos meios que estão disponíveis em cada região. O que não elimina a possibilidade de ocorrência de obstáculos no processo construtivo e organizativo pelas equipes de saúde. Portanto, o apoio matricial necessita ser avaliado com periodicidade para evitar que as barreiras enfraqueçam a forma de operá-lo (SILVA DN, 2011).

Dessa forma, há alguns problemas nas práticas e operacionalização do matriciamento em caráter nacional: fragilização da política nacional de atenção básica, heterogeneidade de concepções e modelos de prática, ausência de suporte das gestões e gerência dos serviços, ausência de periodicidade do processo e pouca interferência na organização dos fluxos na Rede de Atenção Psicossocial, agenda diversas e problemas entre a Estratégia Saúde da Família e equipes apoiadoras, pouca presença do profissional psiquiatra nas ações de apoio matricial, lógica inserida no modelo ambulatorial com foco na prática de encaminhamento e marcação de consultas, bem como a maneira de implantação da proposta em muitas cidades do país, dentre outros. $O$ que foi corroborado pelos estudos investigados na presente investigação (LIMA M e DIMENSTEIN MO, 2016).

Outras barreiras identificadas e reafirmadas são os problemas na compreensão da proposta, do enfoque intensamente biologicista e curativista de alguns profissionais e das próprias impossibilidades para resolução completa das demandas da população. No mesmo contexto, a integração entre os profissionais de saúde permite diálogos conjuntos, o acordo das estratégias, o trabalho interdisciplinar e a elaboração de projetos terapêuticos em comum, esbarrando no forte contingenciamento da demanda (NORDI ABA e ACIOLE GG, 2017).

Em estudo realizado no Rio Grande do Norte evidenciou-se que o matriciamento é uma estratégia relevante na assistência à crise, ao diminuir as chances de ocorrência de momentos de crise ou na abordagem antes que agudize (LIMA M e DIMENSTEIN MO, 2016).

O matriciamento em saúde mental figura várias formas, em razão da realidade em que acontece e das percepções dos atores que o constrói, pois, a forma como é configurado interfere diretamente na sua implementação e desenvolvimento. Em relação ao compartilhamento de saberes entre os diversos núcleos de conhecimento, indica-se o abandono de um processo de trabalho conduzido de maneira individualizada ou fragmentada pela especialização para um trabalho dialogado e interdisciplinar, resultando, inclusive, nos 
atendimentos individuais, expandindo a perspectiva clínica do profissional. Nessa perspectiva, existe um aspecto técnico pedagógico no matriciamento que repercute na modificação do cuidado gerado pelos profissionais com os usuários (JORGE B, et al., 2014).

O avanço e a consolidação do Sistema Único de Saúde vêm se tornando mais que a garantia de uma rede de serviços que ofereça atenção à saúde de qualidade. Tem feito desse modelo um instrumento e espaço de construção de saúde que opera no atendimento das demandas de saúde da sociedade brasileira, pelo processo de enfrentamento dos seus determinantes sociais com a utilização da clínica ampliada, do trabalho em equipe na perspectiva multiprofissional, interativa e participativa, e da construção do cuidado como instrumentos de defesa da vida do indivíduo e da coletividade, o que acarreta a atribuição de que seja tarefa de todos que essa estratégia dê certo, uma proposta relevante na transformação de modelos que objetivam abranger os princípios e diretrizes do sistema de saúde presente no país (NORDI ABA e ACIOLE GG, 2017).

Por fim, o matriciamento pode ser visto como uma estratégia de aproximação dos pontos da rede que servem para possibilitar o cuidado integral ao usuário com transtorno mental, objetivando a corresponsabilização das equipes e o fortalecimento do vínculo entre a atenção básica e os usuários. Frente a essas discussões é necessária monitorização para que essa tecnologia não seja transformada em um ambulatório itinerante de especialidade (CAMPINAS GWS, et al., 2017). Ao enfatizar a relevância do processo dialógico no trabalho em saúde, tanto no nível individual como no coletivo, a nova estratégia facilita a integração das práticas de cuidado em um nível ampliado de atuação.

\section{CONSIDERAÇÕES FINAIS}

$\mathrm{Na}$ análise das práticas de apoio matricial desenvolvidas nos cenários da atenção primária à saúde em caráter nacional, reconhece-se um processo embrionário, ainda a ser intensificado e fortalecido. Mesmo frente às barreiras impostas para o pleno desenvolvimento do matriciamento em saúde mental, ele deve ser fortificado. Nesse sentido, apesar de algumas barreiras impostas, o apoio matricial vem produzindo efeitos significativos para os usuários e na organização dos serviços em relação à assistência em saúde mental. Ressalta-se que o Ministério da Saúde recomenda que a organização da assistência em saúde mental nos serviços de saúde seja baseada no apoio matricial. Assim, é necessário que as equipes de saúde da família fortaleçam e sistematizem espaços de diálogo entre as equipes de apoio matricial e todos os outros atores envolvidos no cuidado em saúde mental.

\section{REFERÊNCIAS}

1. ALMEIDA DL, et al. Política nacional de saúde mental: práticas e desafios na estratégia saúde da família. Temas em saúde, 2019; 19(2): 380-403.

2. BELOTTI M, LAVRADOR MCC. A prática do apoio matricial e os seus efeitos na Atenção Primária à Saúde. Cad. Ter. Ocup, 2016; 24(2): 373-78.

3. BRASIL. Ministério da Saúde. Portaria n. 154 de janeiro de 2008. Cria os Núcleos de Apoio à Saúde da Família NASF. Diário Oficial da União. Brasília, 2008. Disponível em: <http://bvsms.saude.gov.br/bvs/saudelegis/gm/2008/prt0154_24_01_2008.html>. Acesso em: 02 de Fevereiro de 2021.

4. Brasil. Ministério da Saúde. Secretaria de Atenção à Saúde. Departamento de Atenção Básica. Saúde mental: Cadernos de Atenção Básica, no 34. Brasília: Ministério da Saúde, 2013.

5. CAMPOS GWS, et al. Investigação sobre cogestão, apoio institucional e apoio matricial no SUS-São Paulo: Hucitec e Fapesp, 2017.

6. CHIAVERINI DH. Guia prático de matriciamento em saúde mental. Brasília: Ministério da Saúde, 2011.

7. DANTAS FN, PASSOS FCI. Apoio matricial em saúde mental no SUS de Belo Horizonte: Perspectiva dos trabalhadores. Trab. Educ. Saúde, 2018; 16(1): 201-20.

8. FERREIRA TPS, et al. Produção do cuidado em Saúde Mental: desafios para além dos muros institucionais. Interface. $2017 ; 21(61): 373-84$.

9. FORTES S, et al. Psiquiatria no século XXI: transformações a partir da integração com a Atenção Primária pelo matriciamento. Physis, 2014; 24 (4): 1079-1102.

10. GAZIGNATO ECS, SILVA CRC. Saúde mental na atenção básica: o trabalho em rede e o matriciamento em saúde mental na Estratégia de Saúde da Família. Saúde e Debate, 2014; 38 (101): 296-304.

11. GARCIA VDG, et al. Apoio matricial na atenção à saúde mental em uma regional de saúde, Paraná, Brasil. Revista Saúde e Pesquisa, 2017; 10(3): 423-32. 
12. GONÇALVES CR, PERES SR. Matriciamento em saúde mental: obstáculos, caminhos e resultados. SPAGESP Sociedade de Psicoterapias Analíticas Grupais do Estado de São Paulo Revista da SPAGESP, 2018; 19(2): 123-36.

13. IGLESIAS A, AVELLAR LZ. Apoio Matricial: um estudo bibliográfico. Ciência e Saúde Coletiva, $2014 ; 19$ (9): $791-98$.

14. IGLESIAS A, AVELLAR LZ. Matriciamento em Saúde Mental: práticas e concepções trazidas por equipes de referência, matriciadores e gestores. Ciência \& Saúde Coletiva, 2019; 24(4):1247-54.

15. IGLESIAS A, AVELLAR LZ. As Contribuições dos psicólogos para o matriciamento em saúde mental. Psicologia: Ciência e Profissão, 2016; 36(2): 364-79.

16. JORGE MSB, et al. Apoio Matricial: dispositivo para resolução de casos clínicos de saúde mental na Atenção Primária à Saúde. Revista Brasileira de Enfermagem, 2013; 66(5): 738- 44.

17. JORGE B, et al. Possibilidades e desafios do apoio matricial na atenção básica: percepções dos profissionais Psicologia: Teoria e Prática, 2014; 16(2): 63-74.

18. LIMA M, DIMENSTEIN M. O apoio matricial em saúde mental: uma ferramenta apoiadora da atenção à crise. Interface, 2016; 20(58): 625-35.

19. MACHADO DKS, CAMATTA MW. Apoio matricial como ferramenta de articulação entre a saúde mental e a atenção primária à saúde. Cad Saúde Colet. 2013; 21(2):224-32.

20. MENEGAZ JC, et al. O bom professor na área da saúde: uma revisão integrativa da literatura. Sau. \& Transf, 2013; 4(4): 92-9.

21. MENDES KDS, et al. Revisão integrativa: método de pesquisa para a incorporação de evidências na saúde e na enfermagem. Texto Contexto Enferm, 2008;17(4):758-64.

22. NETO MRG, et al. Apoio matricial em saúde mental na percepção dos profissionais especialistas. Aletheia. 2014; 45(1):139-55.

23. NORDI ABA e ACIOLE GG. Apoio matricial: uma experiência da residência multiprofissional em saúde. Trab. Educ. Saúde, 2017; 15(2): 485-500.

24. QUINDERÉ PHD, et al. Acessibilidade e resolubilidade da assistência em saúde mental: a experiência do apoio matricial. Ciênc. Saúde Colet. 2013; 18(7):2157-66.

25. VASCONCELOS MS, et al. Conhecimento de gestores e profissionais da rede de atenção psicossocial sobre matriciamento em saúde mental. Cienc Cuid Saude, 2019; 18(4): 439-50.

26. SALVADOR BD, PIO MAD. Apoio Matricial e Capsi: desafios do cenário na implantação do matriciamento em saúde mental. Saúde Debate, 2016; 40(111): 246-56.

27. SANTOS MA, et al. O matriciamento em saúde mental como dispositivo para a formação e gestão do cuidado em saúde. Physis: Revista de Saúde Coletiva, 2020; 30(4): 1-20.

28. SANTOS LC, et al. Saúde mental na atenção básica: experiência de matriciamento na área rural. Rev Bras Enferm, 2020; 73(1): 201-36.

29. SILVA C et al. A contribuição do apoiador matricial na superação do modelo psiquiátrico tradicional. Psicol. estud, 2019; 24(1): 1-12.

30. SILVA DN. Apoio matricial em saúde mental: uma análise sob ótica dos profissionais de saúde da atenção primária. Revista Portuguesa de Enfermagem de Saúde Mental, 2011; 6(1): 20-7.

31. TREICHEL CAS, et al. Impasses e desafios para consolidação e efetividade do apoio matricial em saúde mental no Brasil. Interface (Botucatu). 2019; 23(1): 180-17.

32. SOUZA MT, et al. Revisão integrativa: o que é e como fazer. Einstein. 2010; 8(1): 102-6.

33. WETZEL C, et al. O matriciamento enquanto dispositivo para o cuidado em saúde mental na estratégia saúde da família. Rev enferm UFPE on line, 2014; 8(6): 1702-8. 